Bayero Journal of Pure and Applied Sciences: 10(1): 489 - 492

ISSN 2006 - 6996

\title{
A SURVEY ON DRUG RELATED PROBLEMS IN CERVICAL CANCER PATIENTS RECEIVING CHEMOTHERAPY IN AHMADU BELLO UNIVERSITY TEACHING HOSPITAL ZARIA
}

\author{
Mustapha, S. ${ }^{* 1}$, Mohammed, M. $^{2}$, Mustapha, L. ${ }^{3}$, Yunusa, I. ${ }^{1}$ and Basgut, B. ${ }^{4}$ \\ ${ }^{1}$ Department of Pharmacology and Therapeutics, Ahmadu Bello University, Zaria \\ ${ }^{2}$ Department of Clinical Pharmacy and Pharmacy Practice, Ahmadu Bello University Zaria \\ ${ }^{3}$ Department of Pharmaceutical and Medicinal Chemistry, Ahmadu Bello University, Zaria \\ ${ }^{4}$ Department of Pharmacology and Clinical Pharmacy, Near East University, North Cyprus \\ *Corresponding Author: +2347061349649; maimunat001@gmail.com
}

\begin{abstract}
A drug-related problem (DRP) is an event or circumstance involving drug therapy that actually or potentially interferes with desired outcome. The complexities in drug regimens used in cervical cancer chemotherapy has led to more serious problems. The aim of this study is to investigate DRPs in cervical cancer patients receiving chemotherapy. The study was a retrospective cross sectional study on patients receiving cervical cancer chemotherapy at Ahmadu Bello University Teaching Hospital Zaria (ABUTH). Patients' medication charts were reviewed from July, 2015 to June, 2016. Data were collected using the Pharmaceutical Care Network Europe (PCNE) Classification of DRP V 6.20. A total of 224 DRPs cases were identified from 65 cervical cancer patients. The DRPs were mostly on treatment effectiveness $(28.1 \%)$, adverse reaction $(29.0 \%)$, and cost $(26.8 \%)$, while the causes were majorly drug selection (28.1\%) and dose selection (29.0\%). The risk factors for the DRPs were found to be comorbidities and polypharmacy. In conclusion, DRPs were common among cervical cancer patients receiving chemotherapy at ABUTH. Pharmacist interventions to achieve a better therapeutic outcome are necessary.

Keywords: Cervical cancer, chemotherapy, drug related problem, pharmacist, polypharmacy,
\end{abstract}

\section{INTRODUCTION}

Cervical cancer is the fourth most common cancer in women, with an estimated 528,000 new cases occurring globally (Ferlay et al., 2013). In Nigeria, it is the second most common female cancer, with an age-standardized incidence rate of 34.5 per 100000 and incidence/ mortality ratio of 0.6 . (Parkin et al., 2003, Jedy-Agba et al., 2012; Ferlay et al., 2013). The global burden of cervical cancer is unevenly distributed throughout the world, with developing countries accounting for over $80 \%$ of all new cases (Ferlay et al., 2010).

Human papillomavirus (HPV) have been known to be involved in the development of more than $90 \%$ of cervical cancer cases (Kumar et al., 2007; Kufe et al., 2009). Other risk factors include birth control pills, having many sexual partners, smoking, starting sex at a young age, and a weak immune system .

In developed countries, the widespread use of cervical screening programs has dramatically reduced rates of cervical cancer (Canavan and Doshi, 2000). Cervical cancer chemotherapy is one of the best approaches to eradicate cancer, but its success is far from satisfactory due to mostly drug related problems.
A drug-related problem (DRP) is an event or circumstance involving drug therapy that actually or potentially interferes with desired outcome (PCNE, 2010). They include adverse drug reactions (ADRs) possibly exacerbated by unnecessary drug therapy, inappropriate choice of drugs, and untreated conditions. An inclusive study of DRPs in cervical cancer patients receiving chemotherapy could provide valuable insights towards prevention and/or management of DRPs. Therefore, the aim of this study was to investigate DRPs in patients receiving cervical cancer chemotherapy in Ahmadu Bello University Teaching Hospital Zaria.

\section{MATERIALS AND METHODS}

Study Design

This is a retrospective cross sectional study on patients receiving cervical cancer chemotherapy at Ahmadu Bello University Teaching Hospital, Zaria. Following approval of the Hospital Research and Ethical Committee (HREC), patients' case files and treatment charts were reviewed for a period of twelve months (July, 2015 to June, 2016). The study was approved by the hospital research ethics committee (HREC). 


\section{Study Population}

All patients who received chemotherapy in the oncology unit, who were diagnosed with cervical cancer, aged 18 and above and who had taken chemotherapy for at least one course within the study period were included in the study. Patients who had not taken chemotherapy for at least six months within the study period were excluded from the study.

Data Collection

Data were collected on potential and manifested DRPs using the Pharmaceutical Care Network Europe Classification V 6.20. (Appendix I). DRPs were identified by crosschecking the data with the standard protocol like Pharmacy Guide to Chemotherapy-Clinical Assessment, drug monographs and drug interaction databases.

\section{Data analysis}

Data were analyzed using the Statistical Package for the Social Sciences (SPSS) version 20.0 software. Pearson chi-square test were used to determine the association between patients factors like age, co-morbidity and number of medications with presence or absence of DRPs. Statistical significance were considered at $p<0.05$.

\section{RESULTS}

The study analyzed a total of 65 female cervical cancer patients, majority of the patients $(27.69 \%)$ fell within 40 - 49 years, with mean age of $48.3 \pm 4.2$ years as shown in figure 1.

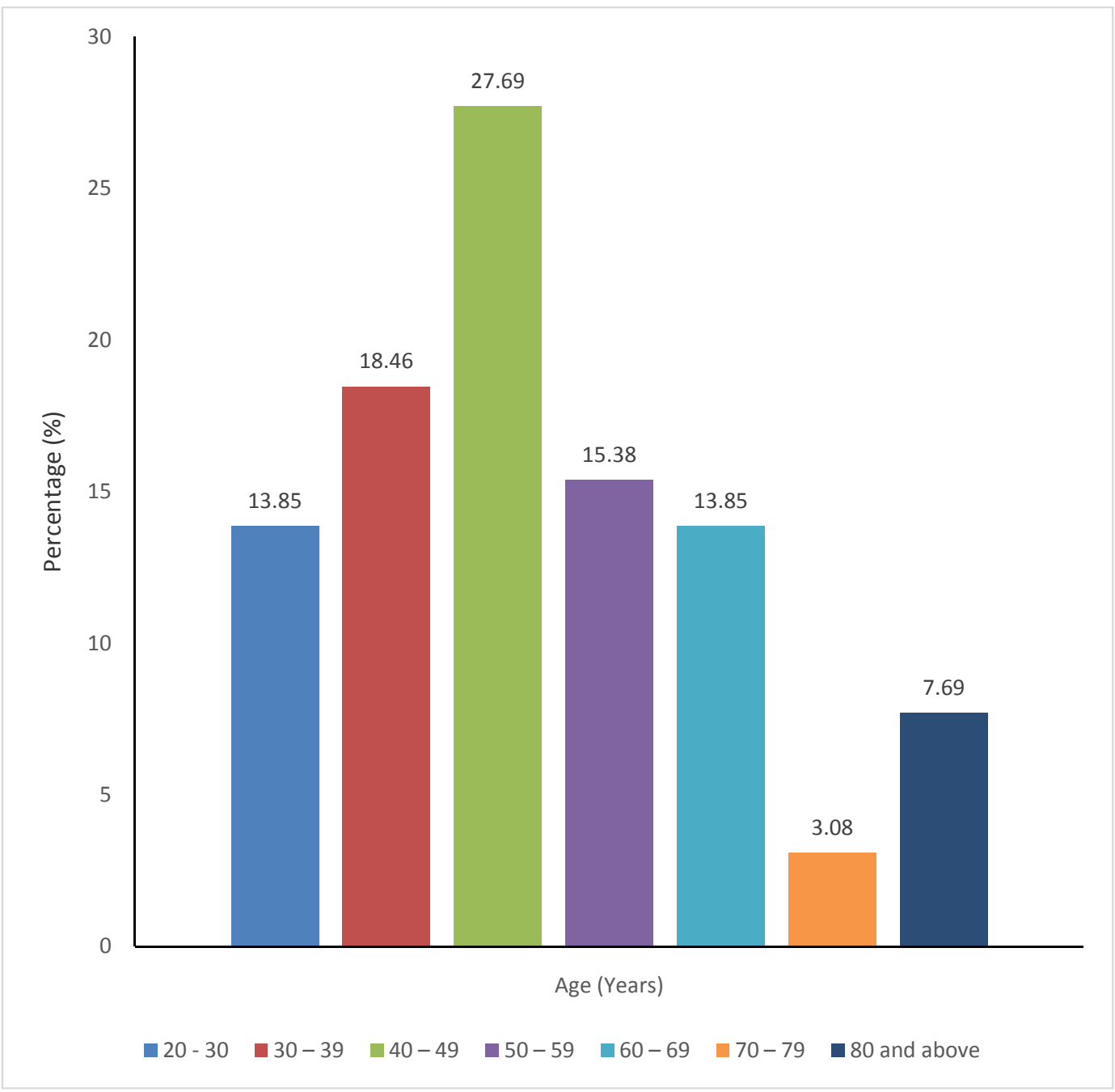

Figure 1: Age distribution of cervical cancer patients attending ABUTH Zaria

Table 1 showed a total of 224 problems were identified in 58 cervical cancer patients with a prevalence of $89.2 \%$. Adverse drug reactions were noted among $29.02 \%$ of the patients, while treatment effectiveness was observed in $28.13 \%$ of the patients. 
Table 2 showed almost all the patients reported nausea and vomiting (46.15\%) then followed by diarrhea $(18.46 \%)$. Table 3 showed there were association $(p<0.05)$ between age, number of medication and comorbidity with presence of DRPs.

Table 1: Types of drug related problems among cervical cancer patients

\begin{tabular}{llll}
\hline S/No. & Type of problem & Freq. & $\begin{array}{l}\text { Percentage } \\
\text { (\%) }\end{array}$ \\
\hline & Treatment effectiveness & 63 & $\mathbf{2 8 . 1 3}$ \\
1. & No effect of drug treatment / therapy failure & 25 & 39.68 \\
2. & Effect of drug treatment not optimal & 18 & 28.57 \\
3. & Wrong effect of drug treatment & 10 & 15.87 \\
4. & Untreated indication & 10 & 15.87 \\
\hline & Adverse reactions & 65 & 29.02 \\
1. & Adverse drug event (non-allergic) & 62 & 95.38 \\
2. & Adverse drug event (allergic) & 3 & 4.62 \\
3. & Toxic adverse drug event & - & - \\
\hline & Treatment costs & 60 & 26.79 \\
1. & Drug treatment more costly than necessary & 51 & 85 \\
2. & Unnecessary drug treatment & 9 & 15 \\
\hline & Others & 36 & 16.07 \\
1. & Patient dissatisfied with therapy & 30 & 83.33 \\
2. & Unclear problem / complaint & 6 & 16.67 \\
\hline
\end{tabular}

Table 2. Adverse reactions reported among cervical cancer patients

\begin{tabular}{lllll}
\hline S/No. & Adverse (ADR) & Suspected Drugs & Frequency & Percentages (\%) \\
\hline 1. & Hypersensitivity & Cisplatin & 3 & 4.62 \\
2. & Nausea and Vomiting & Cisplatin/Paclitaxel/5-FU & 30 & 46.15 \\
3. & Diarrhea & Cisplatin/Paclitaxel & 12 & 18.46 \\
s4. & Loss of hearing & Cisplatin & 3 & 4.62 \\
5. & Mucositis & Cisplatin/5FU/paclitaxel & 6 & 9.23 \\
6. & Seizure & Cisplatin & 2 & 3.08 \\
7. & Loss of hair & Cisplatin/5FU/Paclitaxel & 3 & 4.62 \\
8. & Nephrotoxicity & Cisplatin & 3 & 4.62 \\
9. & Hypotension & Paclitaxel & 3 & 4.62 \\
\hline \multicolumn{4}{l}{}
\end{tabular}

Table 3: Relationship between cervical cancer patients' factors and DRPs

\begin{tabular}{|c|c|c|c|c|}
\hline \multirow[t]{2}{*}{ Patients Factor } & & \multicolumn{2}{|c|}{ Drug Related Problems (DRPs) } & \multirow[t]{2}{*}{$P$ value } \\
\hline & & Yes & No & \\
\hline \multirow[t]{2}{*}{ Age } & $<50$ years & 33 & 6 & 0.006 \\
\hline & $\geq 50$ years & 25 & 1 & \\
\hline \multirow[t]{2}{*}{ Comorbidity } & $<3$ diseases & 53 & 6 & 0.002 \\
\hline & $\geq 3$ diseases & 5 & 1 & \\
\hline \multirow{2}{*}{$\begin{array}{l}\text { Number of } \\
\text { medication }\end{array}$} & $<5$ drugs & 5 & 7 & 0.01 \\
\hline & $\geq 5$ drugs & 53 & 0 & \\
\hline
\end{tabular}

\section{DISCUSSION}

Drug related problems are significant healthcare problems and great proportions are preventable. This study identifies significant cases of cervical cancer. Majority of the women captured in this our study were between the age of 30 and 60 years. This number could be attributed to the wide prevalence of human papilloma virus and lack of its vaccination among developing countries and also due to the fact that women in most developing countries lack access to routine screening (Kumar et al., 2007).

This study identified 224 cases of DRPs in 65 patients receiving cervical cancer chemotherapy. Chemoradiation in cervical cancer patients is among the commonest and best practice in our health institutions and its success is far from satisfactory due to mostly drug related problems. Anticancer drug regimens are administered following established protocols. 
The more complex drug therapy is the higher the risk of experiencing drug related problems (DRPs) such as adverse effects, comorbidities, interactions, medication errors, and nonadherence.

The most common ADRs identified were vomiting, nausea and diarrhea, these events are predictive and have been known to be inherent to the chemotherapeutic drugs. However, recommendations were made in order to avoid serious problems as the case may

\section{REFERENCES}

Ferlay, J., Soerjomataram, I. and Ervik, M. (2013). Cancer Incidence and Mortality Worldwide: IARC Cancer Base No. 11. Lyon, France: International Agency for Research on Cancer. GLOBOCAN 2012 v1.0. http://globocan.iarc.fr

Parkin, D.M., Ferlay, J. and Hamdi-Cherif, M. (2003). Cancer in Africa: epidemiology and prevention. Lyon: IARC Press.

Jedy-Agba, E., Curado, M.P. and Ogunbiyi, O. (2012). Cancer incidence in Nigeria: a report from population-based cancer registries. Cancer Epidemiol. 36:e2718.

Ferlay, J. Shin, H.R. and Bray, F. (2010). Estimates of worldwide burden of be. The risk factors for the DRPs were found to be older age, co-morbidities and polypharmacy. Conclusion

Drug related problems were common among cervical cancer patients receiving chemotherapy at ABUTH with adverse reaction as the most common. Pharmacist recommendation and interventions were necessary to achieve a better therapeutic outcome and improved quality of lives of the cancer patients.

cancer in 2008: GLOBOCAN 2008. Int J Cancer. 127:2893-917.

Kufe, D. (2009). Holland-Frei cancer medicine. (8th ed.). New York: McGraw-Hill Medical. p. 1299. ISBN 9781607950141.

Kumar, V., Abbas, A.K., Fausto, N. and Mitchell, R.N. (2007). Robbins Basic Pathology (8th ed.). Saunders Elsevier. pp. 718-721. ISBN 978-1-4160-2973-1.

Canavan, T.P. and Doshi, N.R. (2000). Cervical cancer". Am Fam Physician. 61 (5): 1369-76. PMID 10735343.

The Pharmaceutical Care Network Europe (PCNE 2010). Classification of Drug Related Problems. Form Version 6.2. PCNE Classification of drug related problems. 